\title{
PROBING THREE- AND FOUR-NUCLEON INTERACTIONS WITH THE DEUTERON BREAKUP REACTION*
}

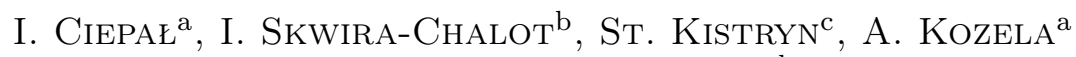
A. MAGIERA ${ }^{\mathrm{c}}$, E. StephaN ${ }^{\mathrm{d}}$

${ }^{a}$ The H. Niewodniczański Institute of Nuclear Physics PAN, Kraków, Poland ${ }^{\mathrm{b}}$ Faculty of Physics, University of Warsaw, Warszawa, Poland

${ }^{c}$ The M. Smoluchowski Inst. of Physics, Jagiellonian University, Kraków, Poland ${ }^{\mathrm{d} I n s t i t u t e}$ of Physics, University of Silesia, Katowice, Poland

(Received December 14, 2016)

Research in the domain of few-nucleon systems concerns reactions involving a small number of nucleons in which observables can be compared directly to exact computational methods - rigorous solutions of the Faddeev equations. The investigations of such systems reveal existence of various dynamical ingredients such as the three-nucleon force, the Coulomb force or relativistic effects. A large set of the cross-section data of the ${ }^{1} \mathrm{H}(d, p p) n$ breakup reaction obtained at energy of $130 \mathrm{MeV}$ was used to trace the Coulomb force effects. Comparisons of the cross-section data with the predictions using the realistic Argonne 18 potential are presented. The new set of invariants was introduced to describe the process with three nucleons in the final state.

DOI:10.5506/APhysPolB.48.481

\section{Introduction}

Deuteron breakup in collision with a proton, leading to a final state of three-nucleon $(3 \mathrm{~N})$ continuum, is one of the simplest processes to study dynamics of few nucleons. The reaction is characterized by a rich kinematics of the final state what makes it selective regarding the applied model of interaction.

In low- and medium-energy domains, the main properties of few-nucleon systems are successfully modeled with the use of the realistic nucleonnucleon $(\mathrm{NN})$ potentials. At intermediate energies, effects of the few-nucleon

* Presented at the Zakopane Conference on Nuclear Physics "Extremes of the Nuclear Landscape", Zakopane, Poland, August 28-September 4, 2016. 
dynamics beyond the pairwise $N N$ interaction start to play the role. Due to availability of exact theoretical calculations and very precise experimental data, it is possible to study variety of dynamical effects, including very subtle Three Nucleon Force $(3 \mathrm{NF})$. In order to correctly describe the system dynamics, calculations should include the model of 3NF (e.g. Tucson Melbourne TM force [1]), the Coulomb force [2,3] and be performed in the relativistic regime [4]. Recently, a significant step forward has been made in including the Coulomb force component into the calculations. It was first attempted within the coupled-channels approach [2] and then applied also in calculations with the Argonne V18 (AV18) + Urbana IX 3NF (UIX) potential [3]. The influence of the Coulomb force on the breakup observables turned out to be quite strong in the very forward angular region [5]. The experimental studies of the breakup process allow one to test the nuclear force structure with possible feedback on the force models.

In the present work, an extensive data set measured at forward angles, is the basis to investigate the effects of the Coulomb force on the breakup cross section. The comparison between the data and theory is presented as a function of four coordinates based on the Lorentz invariants.

\section{Experiment}

The measurement aiming at investigations of the Coulomb force effects in the differential cross section for the ${ }^{1} \mathrm{H}(d, p p) n$ reaction was performed at FZ-Jülich, Germany, with the use of the Germanium Wall (GeWall) setup $[5,6]$. GeWall consisted of three high-purity germanium position sensitive detectors. Two different types of the detectors were used: a thin transmission detector Quirl with a high spatial resolution for determining the position and energy loss of the passing charged particles, and two thick detectors, E1 and E2, for measurement of particle energies with an excellent resolution. The angular acceptance of the apparatus was $5^{\circ}-14^{\circ}$ for the polar and $2 \pi$ for the azimuthal angles. The data collected at the deuteron beam energy of $130 \mathrm{MeV}$ were analyzed for about 112 kinematical configurations (about 1360 cross-section data points), defined by the polar angles of the two outgoing protons, $\theta_{1}, \theta_{2}$, and their relative azimuthal angle $\varphi_{12}$.

\section{Results}

The experimental investigations [5, 6] were focused on a very forward part of the phase space. The studies in this angular range showed that the electromagnetic interaction plays very crucial role in the cross section [5]. In view of this finding, correlations of the observed effects with specific variables are discussed. 
In the previous studies [5], comparisons between the data and theory were performed in terms of the standard variables which define a specific configuration of the two detected breakup protons, i.e. geometry described by combinations of their polar angles, their relative azimuthal angle, and an energy expressed in terms of arc-length along the given kinematics. Such a representation is not practical when one needs to perform a systematic search for dynamical effects over wide energy and phase-space ranges. Therefore, an alternative way of the description of few-body reaction kinematics was proposed in [7]. Mandelstam variables for a two-body reaction were redefined to describe three-body final states and four invariants were introduced:

- the kinetic energy of the relative motion of the proton-proton $\left(E_{\mathrm{rel}}^{p p}\right)$ or the proton-neutron $\left(E_{\mathrm{rel}}^{p n}\right)$ pairs,

- the four-momentum transfer for the nucleon from the entrance channel to the exit channel recalculated to the energy units $\left(E_{\mathrm{tr}}^{p}, E_{\mathrm{tr}}^{n}\right)$.

To search for the electromagnetic force effects, the calculations based on the AV18 potential combined with UIX $3 \mathrm{NF}$ with $(\mathrm{AV} 18+\mathrm{UIX}+\mathrm{C})$ and without (AV18+UIX) Coulomb interaction included were analyzed. To compare the theory with the experimental results, firstly the theoretical predictions were corrected over the detector acceptance and then the ratio $\frac{\sigma_{i}-\sigma_{\mathrm{AV} 18+\mathrm{UIX}}}{\sigma_{\mathrm{AV} 18+\mathrm{UIX}}}$ was calculated, where $\sigma_{i}$ denotes either the experimental datum, or the result of calculations including Coulomb interaction $(\mathrm{AV} 18+\mathrm{UIX}+\mathrm{C})$. In this way, the theoretically predicted effects of the Coulomb force can be compared to the pattern of deficiencies of Coulomb-less calculations in describing the data. The angular acceptance of the GeWall detector corresponds to the region of significant enhancement of the Coulomb force effects in certain regions of the invariants (see Fig. 1) in comparison to the data collected with
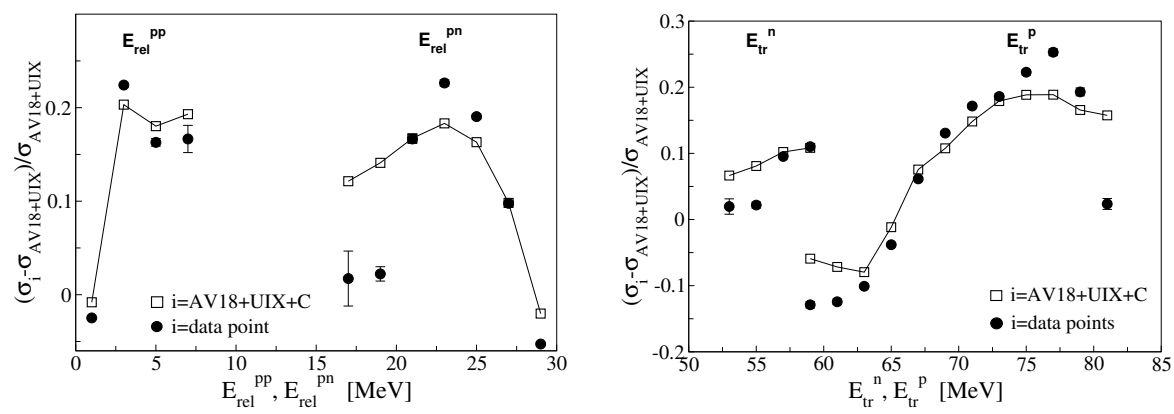

Fig. 1. Net effects of the Coulomb force in the cross section of the $d p$ breakup at 130 $\mathrm{MeV}$ presented as a function of four invariants: $E_{\mathrm{rel}}^{p p}$ and $E_{\mathrm{rel}}^{p n}$ (left panel) and $E_{\mathrm{tr}}^{p}$ and $E_{\mathrm{tr}}^{n}$ (right panel). The coordinates represent the difference between the data and the results of Coulomb-less AV18+UIX calculations (dots) and the predicted Coulomb effect, i.e. the difference between both of the calculations AV18 $+\mathrm{UIX}+\mathrm{C}$ and AV18+UIX (empty squares). 
the SALAD detector, presented in [7]. A very good agreement is observed between the predicted and measured Coulomb effects. In particular, very fast changes of their sign and magnitude at the lowest values of $E_{\text {rel }}^{p p}$ are perfectly reproduced. In this region, two protons are emitted with very similar momenta and the repulsion between them strongly influences the cross section. In the regions of the smallest values of $E_{\text {rel }}^{p n}$ and the largest values of $E_{\mathrm{tr}}^{p}$, quite large differences between the data and theory are seen. They are partially related to the large data uncertainties.

\section{Outlook}

Precise and systematic studies of the breakup reaction in a large part of the phase space $[7,8]$ are very important for understanding of the interaction between nucleons. Currently available theoretical approaches to describe the interaction need very precise and large experimental database to be verified and further developed. To perform cross-energy systematic comparisons of the predictions and the data, the invariant coordinates were introduced [7]. Their usefulness for the studies of net Coulomb effects has been demonstrated.

New experiments to study $3 \mathrm{~N}$ system dynamics in the four-body environment are planned. Such investigations, utilizing a polarized ${ }^{3} \mathrm{He}$ target, are planned at the Cyclotron Center Bronowice of the Institute of Nuclear Physics in Kraków. The studies will allow one to fill a data-deficient sector of $4 \mathrm{~N}$ systems and will trigger performing of the calculations for the scattering observables for such systems in the medium energy region.

\section{REFERENCES}

[1] S.A. Coon, H.K. Han, Few-Body Syst. 30, 131 (2001).

[2] A. Deltuva, A.C. Fonseca, P.U. Sauer, Phys. Rev. C 73, 057001 (2006).

[3] A. Deltuva et al., Phys. Rev. C 80, 064002 (2009).

[4] H. Witała et al., Eur. Phys. J. A 30, 369 (2006).

[5] I. Ciepał et al., Few-Body Syst. 56, 665 (2015).

[6] I. Ciepał et al., Phys. Rev. C 85, 017001 (2012).

[7] E. Stephan et al., Eur. Phys. J. A 49, 36 (2013).

[8] N. Kalantar-Nayestanaki et al., Rep. Prog. Phys. 75, 016301 (2012). 